\title{
The role of seniority-zero states in nuclear level densities
}

\author{
S. Åberg ${ }^{\mathrm{a}}$, B.G. Carlsson ${ }^{\mathrm{a}}$, Th. Døssing ${ }^{\mathrm{b}}$, P. Möller ${ }^{\mathrm{c}}$ \\ ${ }^{a}$ Mathematical Physics, Lund University, P.O. Box 118, S-221 00 Lund, Sweden \\ ${ }^{b}$ Niels Bohr Institute, Blegdamsvej 17, Copenhagen, Denmark \\ ${ }^{c}$ Theoretical Division, Los Alamos National Laboratory, Los Alamos, NM 87545, USA
}

\begin{abstract}
At low excitation energies seniority-zero states dominate the level density of $K=0$ bands in deformed even-even nuclei, while they play no role at higher excitation energies. We describe the level densities in a Fermi-gas model as well as in a combinatorial level-density model and compare to detailed experimental data for some rare-earth nuclei. An explanation is provided for recent observations of an odd-even staggering in the spin-distribution function as an effect of $r$-symmetry of wave functions for deformed nuclei. The structure of $0^{+}$states in deformed nuclei is discussed in the model and compared to data, stressing the role of the seniority quantum number. The Fermigas model is utilized to obtain an overview of the odd-even staggering phenomenon in other mass regions. Odd-even staggering in spherical nuclei, appearing in open-shell nuclei, is briefly discussed as caused by fermion exchange symmetry.
\end{abstract}

Keywords: level-density, seniority-zero states, spin-distribution, $0^{+}$-states

April 2, 2015

\section{Introduction}

With increasing excitation energy, the physics of nuclei gradually changes from being described by discrete states towards more general observables obtained by averaging over several states, such as strength functions and level densities. For heavy nuclei, precise experimental information on level densities based on direct counting of nuclear levels can be carried out at low excitation energy, and then again within a narrow energy window just above the neutron separation energy. A bridge connecting these two regions of precise level-density information is provided by the Oslo method [1] for analyzing $\gamma$-ray spectra from nuclei excited with specific transfer reactions. In the present paper we shall focus on global properties at low excitation energies.

Based on shell-model Monte Carlo calculations recently Alhassid, Liu and Nakada predicted an odd-even staggering in the spin-distribution function for some even-even iron nuclei [2]. In a global study of experimental data, von Egidy and Bucurescu $[3,4]$ could show that the phenomenon appears at low excitation energies for more or less all even-even nuclei. Their careful compilation of level densities was based on counted levels in nuclei measured from the ground state up to a limiting excitation energy of about $1.5 \mathrm{MeV}$. These levels cover a certain angular-momentum interval, typically from angular momentum 0 to 6 (or from $1 / 2$ to 11/2).

Especially, von Egidy and Bucurescu determine two properties of the spin distribution, namely the spin cut-off factor and a parameter which describes the overabundance of states of even angular 
momenta in even-even nuclei, i.e. an odd-even staggering in the spin-distribution function. We shall here discuss how the odd-even staggering phenomenon in deformed nuclei is related to symmetries of the nuclear potential and to the weakening of pair-correlations in few-quasiparticle excitations. The considered symmetry is associated with the $r$-symmetry of a quadrupole deformed potential. For spherical nuclei the effect is caused by the fermion exchange symmetry, that we shall briefly discuss in subsection 5.2 .

The present study mainly concerns deformed nuclei where the odd-even staggering in the spin distribution seems to occur systematically. In quadrupole deformed even-even nuclei, seniority $\mathbf{v}=0$ states give rise to rotational bands with only even angular momenta. We discuss the level density of these states as compared to other states. In Section 2 these two kinds of states are discussed in general statistical terms applying average formulas from a Fermi gas. By applying the combinatorial level-density model described in reference [10] the general structure of these states can be studied more specifically. The level-density model is a straightforward application of thermal degrees of freedom of the folded-Yukawa plus finite-range droplet model, that is well tested for calculations of nuclear masses, fission barriers, ground-state spins and decay rates [5, 6, 7, 8].

In Section 3 we perform a detailed study averaged over the three rare-earth nuclei for which the measured level density from discrete states is most complete. We find that the $\mathbf{v}=0$ states give rise to an odd-even staggering in the spin distribution function at low excitation energies, that disappears at higher excitation energies due to the dominance of $\mathbf{v}>0$ states. To confirm these findings we also study odd-odd and briefly odd-even nuclei, where no $\mathbf{v}=0$ states exist. In Section 4 the structure of $0^{+}$states in well-deformed even-even nuclei is investigated in the model and compared to data. In section 5, the odd-even staggering in other mass regions is addressed in general terms, applying approximate Fermi-gas expressions. Finally, in Section 6 conclusions from the study and a summary of obtained results are provided.

\section{Analytic expressions for a Fermi gas}

First, we consider the intrinsic level density from a single-particle picture in a quadrupole deformed nucleus, determined by a specific energy $E$, neutron number $N$, proton number $Z$ and projection of the angular momentum $K$ on the symmetry axis. Such a level density can be obtained by combinatorial counting $[9,10]$. Alternatively, one can apply a saddle-point procedure, introducing Lagrange multipliers for each of the conserved additive quantities $E, N, Z, K$. For the specific case of a smooth level scheme with equidistant levels, one obtains straightforward analytic expressions valid generally for fermions [11].

In subsection 2.1 we derive a level-density expression for seniority-zero states of even-even nuclei. Assuming a well-deformed shape of the nucleus, we account for the $K$-quantum number in subsection 2.2 , with special attention to seniority $\mathbf{v}=0$ states, which are a special class of $K=0$ states. The total level density including collective rotations is considered in subsection 2.3. In subsection 2.4, the obtained Fermi-gas spin-distribution function is studied for deformed rare-earth nuclei, and is compared to data in subsection 2.5.

\subsection{Single-particle excitations and excitations of pairs}

Figure 1 illustrates an equidistant level scheme, starting with doubly degenerate levels of spacing $\Delta E$ (panel (a)). For evaluating the Fermi-gas level density of all possible many-nucleon excitations, the effective single-particle level spacing is $\Delta E / 2$ (panels (b) and (c)). Finally, panel (d) illustrates 
(a)

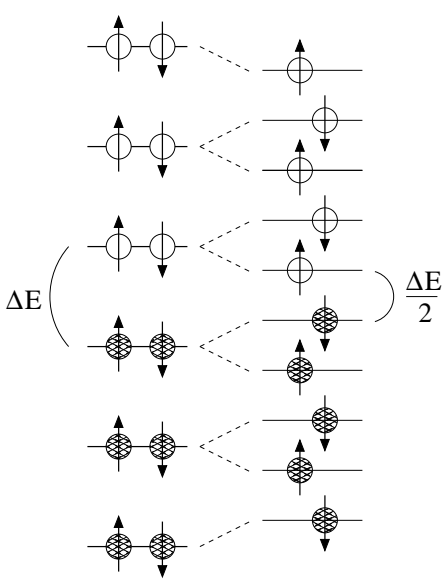

(c)

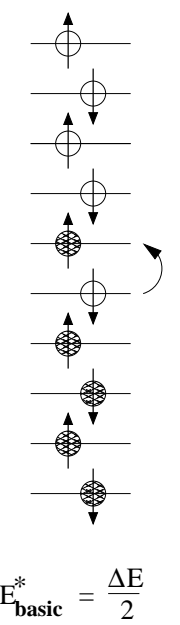

(d)
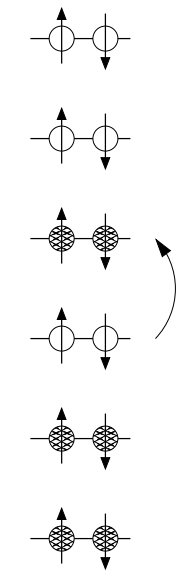

$\mathrm{E}_{\text {basic }}^{*}=2 \Delta \mathrm{E}$

Figure 1: Schematic illustration of an equidistant single particle level scheme, relevant for evaluating Fermi-gas expressions for the level density. Panel (a) displays the doubly degenerate levels of spacing $\Delta E$ in an axially symmetric nuclear potential. Filled and open circles denote occupied or free single-particle states in the ground state of an even particle number. Up- and down arrows symbolize positive and negative projections $\Omega$ of the single-particle angular momentum on the symmetry axis. Considering excited states, each of the states of projection $+\Omega$ and $-\Omega$ can independently be occupied or free, effectively corresponding to single-particle level spacing $\Delta E / 2$, as illustrated in panel (b). In this picture, the spacing $\Delta E / 2$ is the basic excitation energy, as illustrated in panel (c) by the lowest excitation. Considering instead excitations of full pairs of nucleons in time-reversed orbits, relevant for $\mathbf{v}=0$ states, the basic excitation energy becomes $2 \Delta E$, as illustrated in panel (d) by the lowest excitation.

how pairwise excitations (seniority $\mathbf{v}=0$ excitations) carry an energy spacing $2 \Delta E$, that is four times higher than the single-particle spacing. The level-density parameter $a$ is given by [11]:

$$
a=\frac{\pi^{2}}{6} g_{0}=\frac{\pi^{2}}{6} \frac{2}{\Delta E}
$$

where $g_{0}$ is the density of single-particle states. This is for one kind of nucleons. For both protons and neutrons, the level-density parameters add up, and the Fermi-gas expression for the level density reads (rewriting equation (2B-42) of [11]):

$$
\rho(N, Z, E)=\frac{\sqrt{\pi}}{12} \frac{1}{(a E)^{1 / 4}} \frac{1}{E} \exp (2 \sqrt{a E})
$$

The pairwise excitations are characterized by seniority zero, $\mathbf{v}=0$, and for them the level-density parameter is scaled down by a factor of $\frac{1}{4}$, due to the factor 4 on the basic energy spacing:

$$
\rho_{\mathbf{v}=0}(N, Z, E)=\frac{\sqrt{\pi}}{12} \frac{1}{\left(\frac{a}{4} E\right)^{1 / 4}} \frac{1}{E} \exp \left(2 \sqrt{\frac{a}{4} E}\right)
$$

\section{2. $K$ quantum number}

In the axially symmetric deformed potential, the single-particle states are eigenstates of the angular-momentum projection $\Omega$ on the symmetry axis. Each of the doubly degenerate levels con- 
tains projections $+\Omega$ and $-\Omega$. For many-particle many-hole states, the $\Omega$ quantum numbers of excited states sum up to generate the $K$ quantum numbers of excited states, i.e. the projection of the total angular momentum on the symmetry axis. In the limit of many excitations one obtains a Gaussian $K$-distribution, characterized by an effective moment of inertia $\mathcal{J}_{\|}$relative to the symmetry axis. The average energy associated with a given value of $K$ reads $E_{K}=\hbar^{2} K^{2} / 2 \mathcal{J}_{\|}$, and the variance of the $K$-distribution is

$$
\sigma_{K}^{2}=T \mathcal{J}_{\|} / \hbar^{2}
$$

$T$ being the temperature, $T \approx \sqrt{E / a}$, and one obtains a $K$-dependence of the level density in the intrinsic system:

$$
\rho_{\text {intr }}(N, Z, E, K)=\frac{1}{\sqrt{2 \pi} \sigma_{K}} \exp \left(-\frac{K^{2}}{2 \sigma_{K}^{2}}\right) \rho(N, Z, E)
$$

("intr" for intrinsic). The Gaussian factor can subsequently be absorbed back into the intrinsic level density as an energy shift:

$$
\rho_{\text {intr }}(N, Z, E, K)=\frac{1}{12}\left(\frac{\hbar^{2}}{2 \mathcal{J}_{\|}}\right)^{1 / 2}\left[E-\frac{\hbar^{2}}{2 \mathcal{J}_{\|}} K^{2}\right]^{-3 / 2} \exp \left(2 \sqrt{a\left(E-\frac{\hbar^{2}}{2 \mathcal{J}_{\|}} K^{2}\right)}\right)
$$

Taking the Fermi gas model, $\rho_{\text {intr }}$, at face value, the single-particle projections $\Omega$ of the angular momentum, positive as well as negative, are assigned to the levels in a random way, as indicated in Fig. 1 panel (b). This is a good approximation when many excitations are involved. However, it does not take into account that specific states with seniority zero are assigned low energy, caused by the structure of the single-particle level scheme, with time-reversed orbitals carrying the same energy, as indicated on panel (a) of Fig. 1. The seniority-zero states are therefore strongly underestimated by the traditional Fermi-gas expression, Eq.(5), and must be considered separately. Indeed all $\mathbf{v}=0$ excitations carry $K$-quantum number 0 and positive parity, with a level density given by Eq.(2),

$$
\rho_{\mathbf{v}=0}(N, Z, E, K)=\frac{\sqrt{\pi}}{12} \frac{1}{\left(\frac{a}{4} E\right)^{1 / 4}} \frac{1}{E} \exp \left(2 \sqrt{\frac{a}{4} E}\right) \delta_{K 0}
$$

formally denoted by a Kronecker delta.

\subsection{Rotational bands}

In deformed nuclei, each intrinsic state acts as a band-head of a rotational band. The $K$ quantum number refers to the symmetry axis of the potential (the 3 -axis), and in addition we assume that the nuclear shape obeys symmetry with respect to rotations $R_{x}(\pi)$ by an angle $\pi$ around an axis (the 1 -axis) perpendicular to this symmetry axis ( $r$ symmetry). Each intrinsic state with angular-momentum projection $K$ combines in the band head with its $r$-conjugate partner which has projection $-K$. For the special case of the $\mathbf{v}=0$ states, the state and its $r$ - symmetric partner are identical, and only even angular momenta $I$ are possible:

$$
I= \begin{cases}0,2,4, \ldots & \text { for } \mathbf{v}=0 \\ K,(K+1),(K+2), \ldots & \text { for } \mathbf{v}>0\end{cases}
$$

To evaluate the external level density, one takes into account that part of the energy,

$$
E_{\mathrm{rot}}=\frac{\hbar^{2}}{2 \mathcal{J}_{\perp}}\left[I(I+1)-K^{2}\right]
$$


is tied up in the collective rotation and thereby not available for nucleonic excitations. In the extreme single-particle picture implied by the Fermi-gas level density, the rigid-body moment of inertia relative to an axis perpendicular to the symmetry axis should be chosen for the collective moment of inertia, $\mathcal{J}_{\perp}$. This yields

$$
\begin{aligned}
\rho_{\mathrm{ext}}(N, Z, E, I) & =\sum_{K=-I, \cdots, I} \frac{1}{2} \rho_{\mathrm{intr}}\left(N, Z, E-E_{\mathrm{rot}}, K\right) \\
& +\rho_{\mathbf{v}=0}\left(N, Z, E-E_{\mathrm{rot}}, 0\right) \times \bmod (I, 2)
\end{aligned}
$$

("ext" for external). In the first sum, the factor $\frac{1}{2}$ takes into account the fact that each state (including also $K=0$ states), combines with its $r$-symmetric partner to form the band head. In the second line, it is emphasized that the $\mathbf{v}=0$ bands only contribute to even angular momenta $I$. Since the traditional Fermi-gas expression does not specify seniority, equation (9) contains a minor overcounting of $\mathbf{v}=0$ states, that we shall discuss in the next sub-section.

\subsection{Results for the Fermi gas}

Our aim here is to describe rare-earth nuclei, and the Fermi-gas parameters are chosen to represent a nucleus with mass number $A=168$ and deformation $\varepsilon_{2}=0.3$. The level-density parameter is $a=A / 12 \mathrm{MeV}^{1}$. The moments of inertia are chosen as rigid body moments of inertia of a spheriod of radius $1.25 A^{1 / 3} \mathrm{fm}$, leading to the values $\frac{\mathcal{J}_{\text {rig }}}{\hbar^{2}}=0.015 A^{5 / 3} \mathrm{MeV}^{-1}$ for rotation of a sphere and $\mathcal{J}_{\|}=0.9 \mathcal{J}_{\text {rig }}, \mathcal{J}_{\perp}=1.1 \mathcal{J}_{\text {rig }}$ for rotation of the ellipsoid. For comparing to the observed accumulated number of levels as reported in refs. [3, 4], the cumulative level density, i.e. the level density integrated from excitation energy 0 up to a limiting energy, is the relevant quantity.

As a technical detail concerning calculations applying Fermi-gas formulas, one notices that the expressions $(5,6)$ diverge for small values of the energy argument $E$ or $E-E_{\text {rot }}$. This is an artifact of the evaluation of the volume in $N, Z, E, K$-space covered by a grand-canonical ensemble with Lagrange multipliers for these variables [11]. In our calculations, this problem is simply remedied by putting a certain lower limit $E_{\min }$ to the energy $E$ in the denominator, replacing very small values by $E_{\min }$. For rare-earth nuclei, a reasonable value is $0.3 \mathrm{MeV}$ for this minimal value, and the actual value chosen has practically no influence on the results.

In Fig. 2 we compare the (cumulative) density of $K=0$ states specified as $\mathbf{v}=0$ excitations, $\rho_{\mathbf{v}=0}$ in equation (6), with the traditional Fermi-gas expression (5), inserting $K=0$. Values of the accumulated number of states below 1 should be disregarded, so according to the figure, the analytic expressions only become relevant above energy $0.5 \mathrm{MeV}$. Inserting the parameters given above into the expressions $(6,5)$ we obtain for the $A=168$ nucleus:

$$
\begin{gathered}
\rho_{K=0, \mathbf{v}=0}=0.11 E^{-5 / 4} \exp (3.75 \sqrt{E}) \\
\rho_{K=0}=3.5 * 10^{-3} E^{-3 / 2} \exp (7.5 \sqrt{E}) .
\end{gathered}
$$

In Eq.(11) we have written $\rho_{K=0}$, and likewise, there is no mention of seniority in the first line of Eq.(9). However, it would be formally more correct to insert the level density $\rho_{K=0, \mathbf{v}>0}$ with the restriction to $\mathbf{v}>0$. We could write this in terms of a reduction factor $\alpha: \rho_{K=0, \mathbf{v}>0}=\alpha \rho_{K=0}$. At higher excitation energies clearly $\alpha$ approaches unity. In the opposite limit of small excitation 


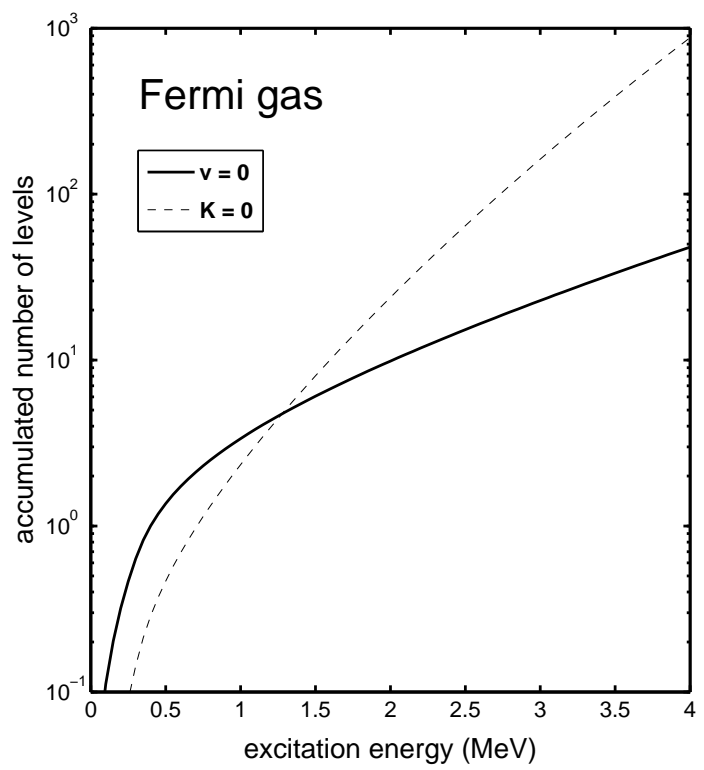

Figure 2: Cumulative level densities obtained by numerical integration of the Fermi-gas expressions with parameters for a nucleus of mass number $A=168$. Solid line: level density of $\mathbf{v}=0, K=0$ states from the here derived expression Eq.(10). Dashed line: level density of $K=0$ bandheads, Eq.(11).

energies we can make an estimate of the size of $\alpha$. The lowest excitations encountered are twoquasiparticle states, and for these one may consider in more detail the distribution of $K=0$ band-heads on seniorities $\mathbf{v}=0$ and $\mathbf{v}=2$. For example, for one-particle-one-hole states, like the one depicted in Fig. 1 (c), suppose that the hole state carries $\Omega=3 / 2$. It will be slightly less probable that the particle state also has $\Omega=3 / 2$, diminishing slightly the probability for such $\mathbf{v}=2$ states to acquire angular momentum projections $K=0$ or $K=3$. Likewise, for $\Omega=5 / 2$, the probabilities for $K=0$ or $K=5$ are diminished. With these arguments, for an oscillator shell, one finds a total of

$$
n_{K=0}=\frac{1}{3}(N+1)\left(N+\frac{3}{2}\right)(N+2)
$$

possible $K=0$ two quasiparticle states, and a number of

$$
n_{K=0, v=0}=\frac{1}{2}(N+1)(N+2)
$$

seniority zero two-quasiparticle states, leading to a factor of

$$
\alpha=1-\frac{n_{v=0}}{n_{\text {total }}}=1-\frac{3}{2 N+3}
$$

. For more than two excited quasiparticles, one obtains

$$
\alpha \approx 1-\left(\frac{3}{2 N+3}\right)^{n_{q u a s} / 2},
$$


where $n_{\text {quas }}$ denotes the number of quasiparticles, since the quasiparticles pair by pair should be in time-reversed states to obtain $\mathbf{v}=0$. Averaging over oscillator shells $\mathrm{N}=5$ for neutrons and $\mathrm{N}$ $=4$ for protons, one finds $\alpha$ values around 0.75 for the lowest excitation energies. Based on this estimate, we conclude that it is a good approximation to ignore the reduction factor, as is done in our calculations. Also, it is important to point out that in the combinatorial treatment of the level density this problem will not appear since all states are identified with exact seniority.

Addressing now in more detail the calculated level density (9) with parameters for mass number $A=168$, Fig. 3 shows the cumulative level density as a function of angular momentum for the Fermi gas. Due to the presence of bands built on $\mathbf{v}=0$ states, a clear odd-even staggering appears in the spin distribution function. With increasing excitation energy, the level density of $\mathbf{v}>0$ states increases much faster than that of $\mathbf{v}=0$ states, and the odd-even staggering is seen to gradually disappear.

\subsection{Even-even nuclei, comparison to data}

Already this simple Fermi-gas model may be compared to experimental data. For rare-earth nuclei, the most complete set of levels at low excitation energy are recorded for the nuclei ${ }^{156} \mathrm{Gd}$,

${ }^{158} \mathrm{Gd}$ and ${ }^{168} \mathrm{Er}$ [4], being fairly complete up to around $2.1 \mathrm{MeV}$ of excitation energy, and up to around 6 units of angular momentum. Table 1 gives the information concerning number of states within the relevant energy and angular momentum intervals.

\begin{tabular}{|c|c|c|c||c|} 
even-even & \multicolumn{4}{|c|}{} \\
\hline Nucleus & $\begin{array}{c}\text { Energy } \\
(\mathrm{MeV})\end{array}$ & $\begin{array}{c}\text { Spin } \\
(\hbar)\end{array}$ & $\begin{array}{c}\text { Number } \\
\text { of states }\end{array}$ & $\begin{array}{c}\text { deformation } \\
\left(\varepsilon_{2}, \varepsilon_{4}, \varepsilon_{6}\right)\end{array}$ \\
\hline${ }^{156} \mathrm{Gd}$ & $0-2.10$ & $0-6$ & 65 & $(0.250,-0.047,0.023)$ \\
${ }^{158} \mathrm{Gd}$ & $0-2.10$ & $0-6$ & 59 & $(0.250,-0.040,0.027)$ \\
${ }^{168} \mathrm{Er}$ & $0-2.10$ & $0-6$ & 68 & $(0.250,-0.033,0.018)$ \\
\hline
\end{tabular}

Table 1: Overview of the data for the three even-even nuclei. Given are the intervals in energy and angular momentum and presently applied, as well as the number of states within these intervals. The last column gives the deformation of the folded Yukawa potential that we shall apply for the combinatorial level density.

However, it should be kept in mind that up to the energy $2.1 \mathrm{MeV}$ on the average 5 levels in each nucleus, typically found in transfer or pickup reactions, have not been assigned angular momentum and parity. Also, it seems that the reactions studied are somewhat selective with respect to the band-head angular momentum. For the three nuclei, only one band-head of $K=6$ is (tentatively) assigned, and none with $K=5$ or $K>6$. We shall evaluate the odd-even staggering as a function of excitation energy, and with averaging over the three nuclei. This makes the results more robust with respect to the incompleteness of the experimental information.

Figure 3 includes the experimental data of the average number of accumulated levels for the three nuclei shown as the thick red circles connected by solid lines. First of all, one sees that the counted number of levels displays an odd-even staggering, both in experiment and resulting from the Fermi gas calculations of the cumulative level density. In the calculations the odd-even staggering is caused by the bands built on the $\mathbf{v}=0$ intrinsic states, and the effect is gradually decreasing with increasing excitation energy. Keeping in mind that the experiment is uncertain 


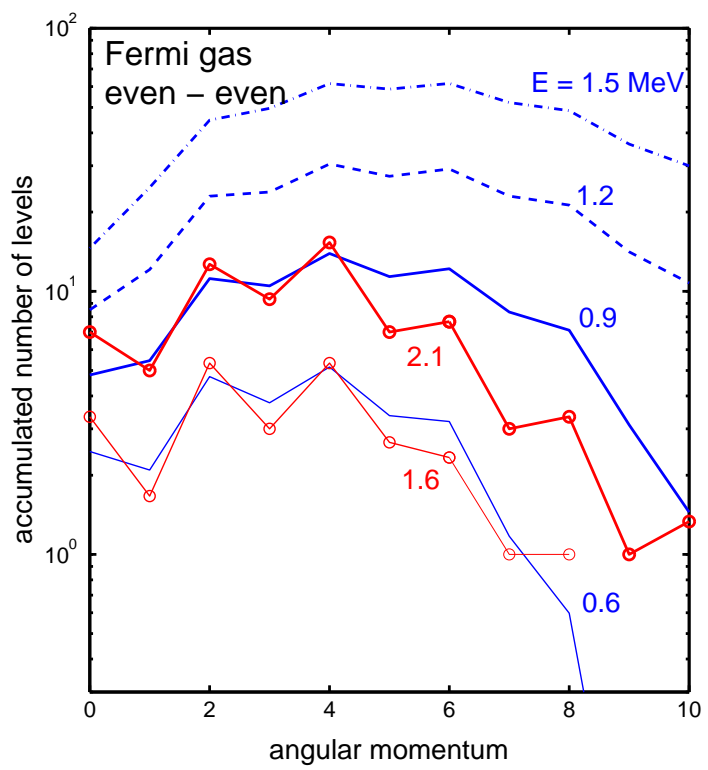

Figure 3: Experimental spin-distribution function is compared to Fermi-gas spin distributions accounting for $\mathbf{v}=0$ states. The experimental value of the average number of counted levels as function of angular momentum for the three nuclei in Table 1 is shown together with the accumulated number of levels obtained from the Fermi-gas level density. The parameters of the Fermi-gas level density are selected to represent a nucleus with mass number 168. The number of levels are shown for the different upper energy limits 0.6 (solid thin blue line), 0.9 (solid thick blue line), 1.2 (dashed blue line) and $1.5 \mathrm{MeV}$ (dot-dashed blue line) for the Fermi-gas model, and for the energy limits 1.6 (thin red circles) and $2.1 \mathrm{MeV}$ (thick red circles) for experimental data. Notice the log-scale of the ordinate.

above angular momentum 6, one sees in Fig. 3 that the best agreement with the experiments is obtained with counted levels of the Fermi gas up to $0.9 \mathrm{MeV}$. This is considerably smaller than the experimental upper limit of $2.1 \mathrm{MeV}$. Similarly, experimental states up to $1.6 \mathrm{MeV}$ are seen to be well described by the Fermi gas with the energy limit set to $0.6 \mathrm{MeV}$. We attribute this energy difference to the pairing, which generates a much smaller level density at low excitation energy (as well as a backshift of the level density further up in excitation energy).

Figure 4 displays how the different values of the band-head $K$-quantum numbers contribute to the accumulative external level density. Neglecting the $\mathbf{v}=0$ level density, there are about twice as many bandheads with $K=$ than with $K=0$. This yields approximately 3 times as many levels of angular momentum $I=1$ as compared to $I=0$, that is a rough proportionality with $2 I+1$ for the level density at small angular momenta, as displayed by the thin solid line in Fig. 4. For angular momentum $I=3$ and up, the number of states deviate from the $2 I+1$ dependence and starts to be reduced due to the increasing energy needed to form states with high $I$. This is described by a spin cut-off factor that we shall return to below. In total, the $\mathbf{v}>0$ states give rise to a smoothly varying spin distribution (thin solid line). Adding now the $\mathbf{v}=0$ levels, that give rise to even spin-value states only, one sees that for the small excitation energies in question, the smooth spin-distribution function is overlayed with an odd-even staggering (thick solid line). 


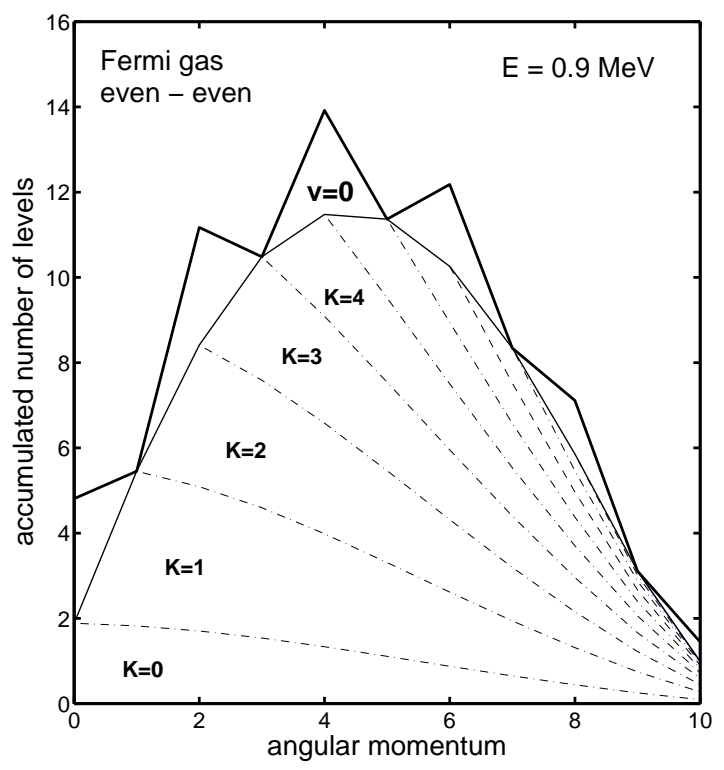

Figure 4: The distribution of $K$ quantum numbers in the accumulated Fermi-gas level density for upper energy 0.9 $\mathrm{MeV}$. The intervals between dot-dashed lines are denoted by the value of the $K$ quantum number, and their sum is given by the thin solid line. In addition to these states come the $\mathbf{v}=0$ states, which only contribute to the even angular momenta, eventually yielding the total number of accumulated levels, denoted by a thick solid line, and carrying an odd-even staggering

\section{Combinatorial level density with blocking BCS}

For the low excitation energies here studied nuclear structure features play an important role. It is therefore important to go beyond the Fermi-gas model and consider a microscopic level-density model, and we shall apply the combinatorial level-density model presented in [10]. The singleparticle levels are taken as eigenstates of the folded Yukawa potential, as determined by Möller et. al. [6].

Pairing drastically reduces the level density at low excitation energies. Presently, we include pairing as described in reference [10]. For each many-particle-many-hole configuration, the BCS equations are solved, taking into account blocking of excited quasiparticle states. Thus, for example, the configuration (c) of Fig. 1 is a two-quasiparticle configuration with two of the doubly degenerate levels blocked to the pairing interaction. Configuration (d) of Fig. 1 is a two-quasiparticle level, with quasiparticles in two time-reversed states. In some respects, this corresponds to a double blocking of the corresponding doubly degenerate level. The BCS equations are solved simultaneously for the pair-gap and the Fermi energy for each configuration.

All many-quasiparticle excitations in the considered energy region are considered. The spinprojections on the symmetry axis of the excited quasiparticles are properly coupled to all allowed $K$-values, and each $K$-state is considered as a band head. Subsequently, a rotational band, see Eqs. $(6,7)$, is built on each band head, where the moment of inertia is taken as the rigid-body moment of inertia times a reduction factor depending on the neutron- and proton pair-gaps, determined for 
each band head [12]. The reduction factor is determined from Belyaevs cranking moment of inertia by averaging over contributing orbits (cf Ref. [13]).

This combinatorial model was set up to primarily describe level densities [10]. Indeed, it provides a detailed microscopic description of individual nuclear states, from the ground-state region to the neutron-separation region. However, since it does not include correlations beyond BCS pairing, no neutron-proton interaction, or any rotational coupling, some detailed properties of the here studied low-lying states may be missing. Assuming that the gross structure is described by the model, we shall therefore (usually) average over several states, and also average over a few nuclei with similar properties when comparing to low-energy data. Detailed rearrangements of levels due to the not included interactions will then be smeared out by the averaging.

In subsection 3.1 results from the combinatorial level-density model are compared to data for the previously considered three rare-earth even-even nuclei. Spin cut-off and staggering parameters are extracted and compared to data in subsection 3.2. In subsection 3.3 the theory is applied to odd-odd nuclei.

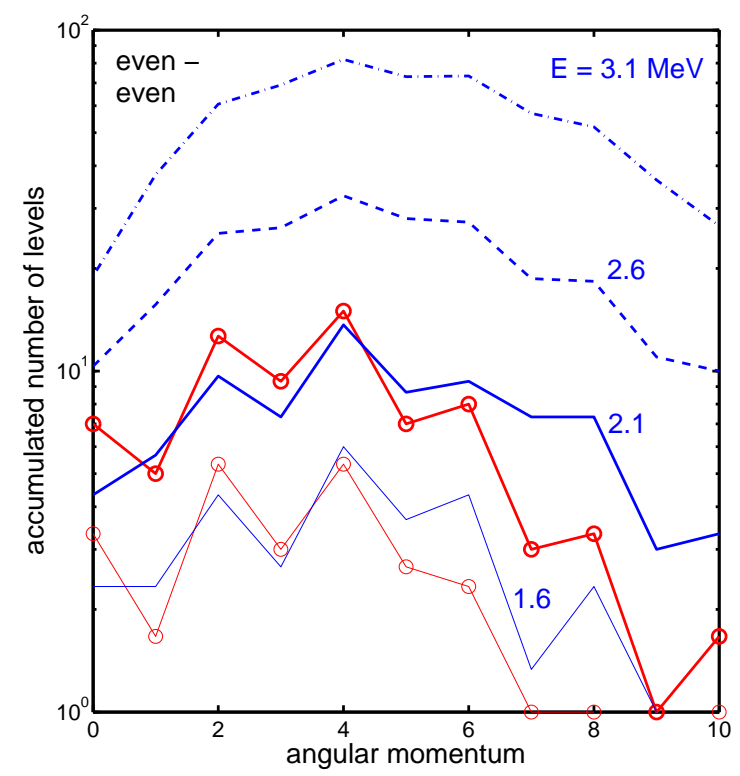

Figure 5: Same as Fig. 3, now for the combinatorial level density based on single-particle energies from the folded Yukawa potential, including pairing, and averaging over the three even-even nuclei shown in Table 1. Notations similar to Fig. 3, but the four calculated curves (blue curves) now correspond to the energy limits 1.6, 2.1, 2.6 and $3.1 \mathrm{MeV}$, respectively.

\subsection{Results for the combinatorial level density}

In Fig. 5 we show the accumulated number of levels from the combinatorial level-density model [10], compared to the experimental data. For both limiting energies, $1.6 \mathrm{MeV}$ and $2.1 \mathrm{MeV}$, a fair agreement is obtained. In order to have sufficient statistics for comparison to data, the energies 1.6 $\mathrm{MeV}$ and $2.1 \mathrm{MeV}$ seem appropriate. Up to $1.6 \mathrm{MeV}$, the experimental level scheme is almost 
complete. In the energy interval between $1.6 \mathrm{MeV}$ and $2.1 \mathrm{MeV}$, surely some levels are missing, probably mostly at the higher angular momenta. Above $2.1 \mathrm{MeV}$ many levels are missing, and it becomes increasingly difficult to identify bands, probably because the onset of mixing of rotational bands takes place around this energy [15]. From the combinatorial level density model, it is seen how the staggering smoothly decreases with increasing excitation energy (as was also seen in the Fermi-gas model) and has almost disappeared at $3.1 \mathrm{MeV}$. This is because the fraction of $\mathbf{v}=0$ states decreases with increasing excitation energy.

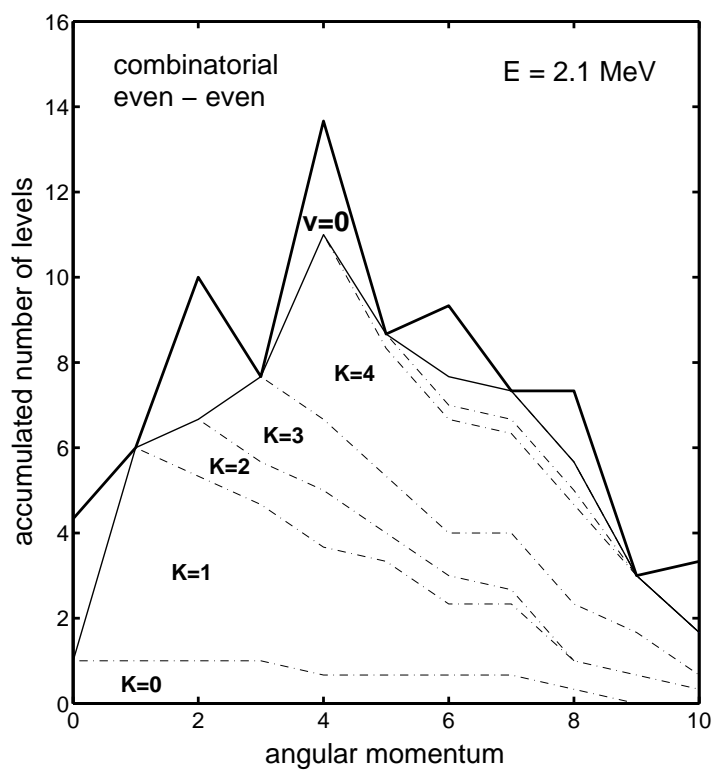

Figure 6: Same as Fig. 4, now for the combinatorial level density, including all states up to an upper energy of 2.1 $\mathrm{MeV}$.

Figure 6 shows the distribution of the levels on the $K$-quantum numbers obtained from the combinatorial model. There is a marked difference to the smooth behavior displayed by Fermi gas results on Fig. 4. In the nuclear potential valid for the three considered nuclei, there is an overabundance of single-particle levels with $\Omega=\frac{3}{2}$ and $\Omega=\frac{5}{2}$ around the Fermi surface both for protons and neutrons. Especially, one finds levels with these values of $\Omega$ as pseudospin partners as well as projections of high- $j$ orbitals. This gives rise to $K=1$ and $K=4$ states, which are seen to yield the major contribution to the cumulative level density.

This dominance of $K=1$ and $K=4$ is only partially supported by the data, displayed in Fig. 7. Especially, $K=2$ states are more abundant than predicted by the combinatorial level density for these three nuclei, as is seen by comparing the areas covered by $K=2$ states in Figs. 6 and 7. In the data, additional levels not placed in bands occur, labeled "non-band levels" in the figure. They are fairly evenly spread over all angular momenta, and their presence does not affect the present conclusions. The accumulated number of all levels up to a given excitation energy and up to angular momentum 6 is shown in Fig. 8 for the three considered nuclei. We extend the inclusion of experimental levels from the Brookhaven database [14] beyond $2.1 \mathrm{MeV}$ to include all 


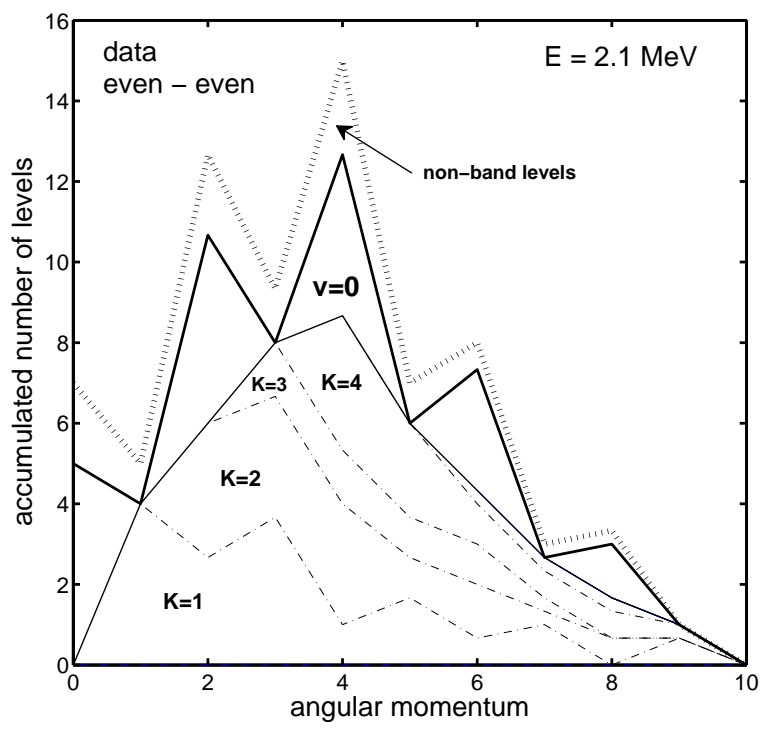

Figure 7: Same as Figs. 4, and 6, now for the experimental levels of Table 1.

levels labeled by parity and angular momentum (also tentative assignments in parenthesis). Also states of assigned parity, but with two or three possible angular momenta are included, making in each case a random choice of the angular momentum. The data is compared to both the Fermi-gas model and the combinatorial model. A remarkably good agreement between the combinatorial level density and the data is seen up to around $2.3 \mathrm{MeV}$, beyond which energy the missing experimental information is apparent. The Fermi-gas model under estimates the level density at low excitation energies, and cannot describe the low-energy structural behavior. The Fermi-gas model has been backshifted by $1.2 \mathrm{MeV}$, which corresponds to the excitation energy of the lowest 2-quasiparticle states in the combinatorial level density. At this excitation energy the density of states is indeed seen to suddenly increase both in the combinatorial model and in data, signaling the opening up of 2-quasiparticle excitations.

\subsection{Spin cut-off and staggering parameters}

To describe the experimental curves of accumulated number of levels $N(I)$ versus angular momentum, von Egidy and Bucurescu [3, 4] introduced a parameterization consisting of a spin cut-off function times an odd-even staggering:

$$
N_{\mathrm{sc}}(I)=(2 I+1) N_{0} \exp \left(-\frac{I(I+1)}{2 \sigma^{2}}\right) \begin{cases}\left(1+(-1)^{I} \delta\right) & \text { for } I \neq 0 \\ \left(1+\delta_{0}\right) & \text { for } I=0\end{cases}
$$

Here, the factor $(2 I+1)$ is a phase-space factor, and the spin cut-off factor $\exp \left(-\frac{I(I+1)}{2 \sigma^{2}}\right)$ is related to the energy tied up in the angular momentum, cf. equations $(5,8,9)$ for the Fermi-gas expressions. The parameter $\delta$ measures the amount of even-odd staggering in the spin-distribution, and especially $\delta_{0}$ is a measure of the over-abundance of $I=0$ states. For a given function $N(I)$ 


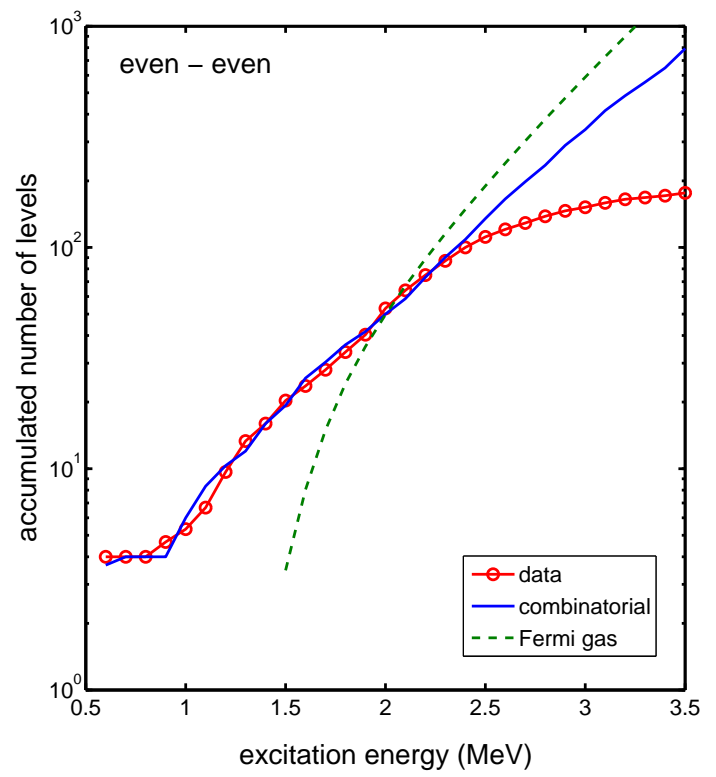

Figure 8: The average accumulated number of levels in the three nuclei (Table 1) for all levels with angular momentum up to 6 as function of excitation energy is shown for (i) the data (solid red line with circles) (ii) the combinatorial level-density model (blue solid line) (iii) the Fermi gas - with the energy scale displaced by $1.2 \mathrm{MeV}$ (green dashed line). The data are incomplete above $2.1 \mathrm{MeV}$.

obtained from experimental data, by combinatorial counting, or by energy integration for the case of the Fermi gas, the parameters $\sigma^{2}$ and $\delta$ are determined to give the best $\mathrm{ft}$ to $N(I)$ within the interval from $I=1$ up to an including a maximum of $I_{\max }=6$. The parameter $\delta_{0}$ is subsequently determined for $I=0$ states only.

In Fig. 9 we show the staggering parameters, $\delta$ and $\delta_{0}$ (left-hand part) as well as the spin cut-off parameter, $\sigma^{2}$ (right-hand part) as deduced from data, from the combinatorial and from the Fermigas model. For excitation energies below about $1 \mathrm{MeV}$, only the ground band is found for these even-even nuclei, cfr. Fig. 8, and for this one band, the parameterization Eq.(12) is quite artificial. According to the present results, the two staggering parameters $\delta_{0}$ and $\delta$ are both measures of the amount of bands built of $\mathbf{v}=0$ states relative to all bands built on $\mathbf{v}>0$ states. With increasing angular momentum, the latter becomes more abundant, roughly with the factor $2 I+1$. One then expects that the ratio of $\delta_{0}$ to $\delta$ should approximately be determined by this factor:

$$
\delta_{0} / \delta \approx 2\langle I\rangle+1=I_{\max }+2
$$

where $\langle I\rangle=\left(I_{\max }+1\right) / 2$ is the midpoint of the interval in angular momentum from $I=1$ to $I_{\max }$ over which $\delta$ is determined. With $I_{\max }=6$ we get the estimate $\delta_{0} / \delta \approx 8$, that is confirmed by the result shown in Fig. 9.

The overall decrease with excitation energy of the two staggering parameters obtained from data is well described in the combinatorial model. The odd-even staggering in the spin-distribution function is strong at low excitation energies, while it has more or less disappeared at $3 \mathrm{MeV}$ excitation energy. It is interesting to note that also the structureless Fermi-gas model, extended by 


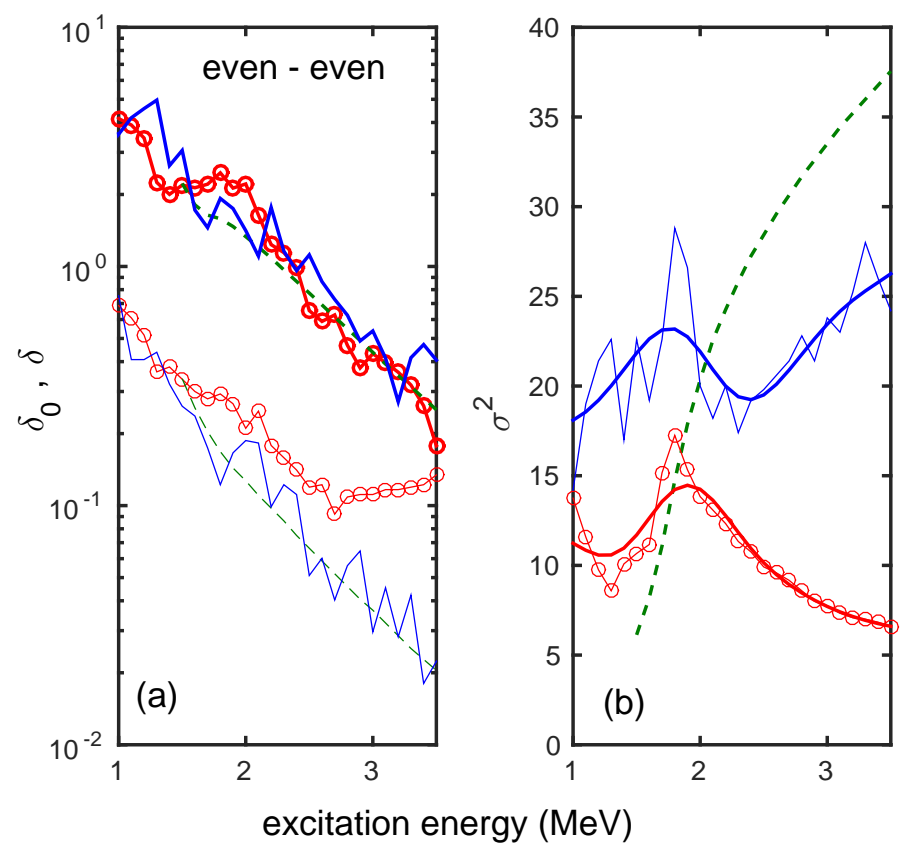

Figure 9: The staggering parameters, $\delta$ and $\delta_{0}$ (a) and the spin cut-off parameter, $\sigma^{2}$ (b) versus excitation energy. The three parameters are fitted to all levels with angular momentum up to 6 for (i) the data of Table 1 (red lines with circles), (ii) the combinatorial levels (blue solid lines), (iii) the Fermi gas (energy displaced by 1.2 MeV; green dashed lines). Results are shown for values of energy 1.0, 1.1, . MeV with spacing $0.1 \mathrm{MeV}$. In panel (a) the three lower curves refer to $\delta$ and the three upper curves to $\delta_{0}$. In panel (b), thick solid red and blue lines show values of $\sigma^{2}$ smoothed over energy intervals of dispersion $0.4 \mathrm{MeV}$. The data are incomplete above $2.1 \mathrm{MeV}$.

the $\mathbf{v}=0$ part, provides a good description of this behavior, providing a backshift of the energy is introduced, cf. Fig. 3.

A more detailed inspection of Fig. 9 shows that within the excitation energy interval from 1.6 $\mathrm{MeV}$ to $2.1 \mathrm{MeV}$, where the data sets are still rather complete, the experimental staggering parameter $\delta$ is about a factor of 1.5 larger than calculated by the combinatorial model. This is also what Fig. 5 shows: the odd-even staggering is somewhat stronger in the data than in the calculations. Above approximately $2.5 \mathrm{MeV}$, the data are dominated by $0^{+}$and $2^{+}$states determined in pair transfer reactions, leading to artificially high values of $\delta$.

The spin cut-off parameter, panel (b) of Fig. 9, is the product of an effective moment of inertia times an effective temperature, $\sigma^{2}=T \mathcal{J}_{\text {eff }} / \hbar^{2}$, cf. equation (3). For accumulated levels, both moment of inertia and temperature should contain averaging over excitation energy, roughly referring to the maximum energy minus the temperature. For the deformed nuclei in question, the effective moment of inertia is some average of the perpendicular moment of inertia and the parallel moment of inertia, both of which are affected by pairing in our calculations. Within the energy interval from $1 \mathrm{MeV}$ to about $2.1 \mathrm{MeV}$, where the data sets are quite complete, the combinatorial model overestimates the value of $\sigma^{2}$ by a factor of about 1.7. This may be caused by a general overstimate of the collective moment of inertia for the bands based on two-quasiparticle excitations, but a systematic investigation of this has not been carried out. The non-monotonic behavior of $\sigma^{2}$, displaying a maximum around excitation energy $1.9 \mathrm{MeV}$ both in data and calculations, is caused 
by the appearence of several low-K bandheads around this energy.

\subsection{Odd-odd nuclei}

For odd-odd nuclei the ground state typically has seniority $\mathbf{v}=2$, and no $\mathbf{v}=0$ states exist in the spectrum. In the Fermi-gas model, there is thus no second line in equation (9); all bands contain even as well as odd values of the angular momentum. Consequently, we do not expect any odd-even staggering for odd-odd nuclei, provided the odd-even staggering in the spin-distribution function for deformed even-even nuclei is caused by the seniority-zero states with $K=0$ (as proposed above).

The combinatorial model can readily be applied also to odd-odd nuclei. In doing this, specific structural properties of the states in odd-odd nuclei are however ignored, in particular effects from the neutron-proton interaction. Also signature splitting between rotational band partners is ignored in the present simple model. In the applied single (quasi)-particle model, it is conjectured that such effects sometimes shift up the energy of levels, sometimes shift them down, in such a way that their effects are averaged out when adding up the number of states. The exact energy of individual levels is therefore not expected to be reliable, but, rather quantities averaged over several states and nuclei. We therefore select a few well-deformed odd-odd nuclei and average over their low-energy spin-distributions.

Guided by the tables in reference [4], we select the three nuclei ${ }^{166} \mathrm{Ho},{ }^{176} \mathrm{Lu}$ and ${ }^{182} \mathrm{Ta}$ as nuclei with rather complete level schemes up to excitation energies of around $0.8 \mathrm{MeV}$. (Still, the data for ${ }^{182} \mathrm{Ta}$ are somewhat incomplete for angular momenta 0 and 1 , according to reference [4]).

\begin{tabular}{|c|c|c|c||c|}
\hline odd-odd & \multicolumn{5}{|c|}{} \\
\hline Nucleus & $\begin{array}{c}\text { Energy } \\
(\mathrm{MeV})\end{array}$ & $\begin{array}{c}\text { Spin } \\
(\hbar)\end{array}$ & $\begin{array}{c}\text { Number } \\
\text { of states }\end{array}$ & $\begin{array}{c}\text { deformation } \\
\left(\varepsilon_{2}, \varepsilon_{4}, \varepsilon_{6}\right)\end{array}$ \\
\hline${ }^{166} \mathrm{Ho}$ & $0-0.8$ & $0-6$ & 66 & $(0.267,0.020,0.021)$ \\
${ }^{176} \mathrm{Lu}$ & $0-0.8$ & $0-6$ & 41 & $(0.250,0.080,-0.012)$ \\
${ }^{182} \mathrm{Ta}$ & $0-0.8$ & $0-6$ & 46 & $(0.233,0.100,-0.031)$ \\
\hline
\end{tabular}

Table 2: Overview of the data for the three odd-odd nuclei. Given are the intervals in energy and angular momentum and presently applied, as well as the number of states within these intervals. The last column gives the deformation of the folded Yukawa potential that we apply for the combinatorial level density.

Figure 10 shows the accumulated number of levels as function of angular momentum for the two upper energy limits $0.5 \mathrm{MeV}$ and $0.8 \mathrm{MeV}$ in the data, together with calculations for upper energy limits $0.5,0.8,1.1$ and $1.4 \mathrm{MeV}$. Neither data nor calculations display any indication of an odd-even staggering. This supports the previous explanation that $\mathbf{v}=0$ states are the origin of the systematic odd-even staggering in the spin-distribution function.

The absence of odd-even staggering can be quantitatively expressed by applying the same fits of spin cut-off functions with staggering (12) as was done for even-even nuclei. The result is shown in Fig. 11. In contrast to the systematic behavior seen for even-even nuclei, the staggering for odd-odd nuclei seems to appear as a random noise, displaying values which are about a factor of 10 smaller. The right-hand frame in Fig. 11 shows the spin cut-off parameter. Increasing deviation between calculation and data is caused by missing experimental levels. 


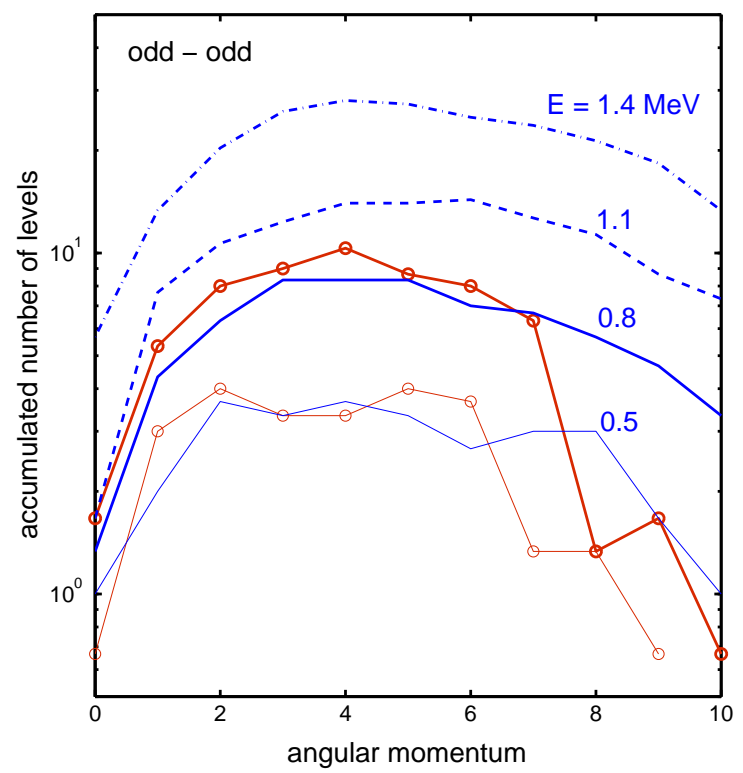

Figure 10: Same as Fig. 5, now for the three odd-odd nuclei of Table 2. For the data, the upper energy limits are $0.5 \mathrm{MeV}$ (red thin lines with circles) and $0.8 \mathrm{MeV}$ (red thick lines with circles). For the combinatorial level density the upper energy limits are in ascending order $0.5,0.8,1.1$ and $1.4 \mathrm{MeV}$.

For completeness, Fig. 12 shows the accumulated number of levels as function of excitation energy for the three odd-odd nuclei. Keeping in mind that the data are complete up to around $0.8 \mathrm{MeV}$, a satisfactory agreement is found. For the odd-odd nuclei, the states of lowest energies are 2-quasiparticle excitations, with one quasiparticle in the neutron system and one in the proton system. Some pairing is remaining with pairing gaps of the order of $0.4 \mathrm{MeV}$, compared to about $0.9 \mathrm{MeV}$ for the even-even nuclei. Consequently, it costs some energy to break further pairs. The lowest four quasiparticle states have an energy around $1.0 \mathrm{MeV}$, and four-quasiparticle states start to dominate the level density from about $1.5 \mathrm{MeV}$. The cumulative level density for the even-even nuclei shown in Fig. 8, displays a similar structure. For the even-even nuclei the ground state band is on its own up to around $1.1 \mathrm{MeV}$, where 2-quasiparticle states enter. At around $2.2 \mathrm{MeV}$ 4-quasiparticle states enter and they dominate the level density around $3.0 \mathrm{MeV}$. This gives rise to a bumpy structure of the level density, with a small but significant increase of the temperature just below the energy where the 4-quasiparticle states set in. This effect is described in reference [16]. A bumpy structure of the level density, maybe signaling the onset of 2-quasiparticle and 4-quasiparticle states, has been observed by the Oslo group [17].

\subsection{Odd-mass nuclei}

With regard to staggering, odd-mass nuclei are similar to odd-odd nuclei. All angular momenta $I=K, K+1 \cdots$ may be generated on top of each bandhead of a given $K$ quantum number. Considering the situation in more detail, all rotational bands may carry a signature splitting, which for some nuclei potentially could lead to a staggering in the level densities as function of angular 


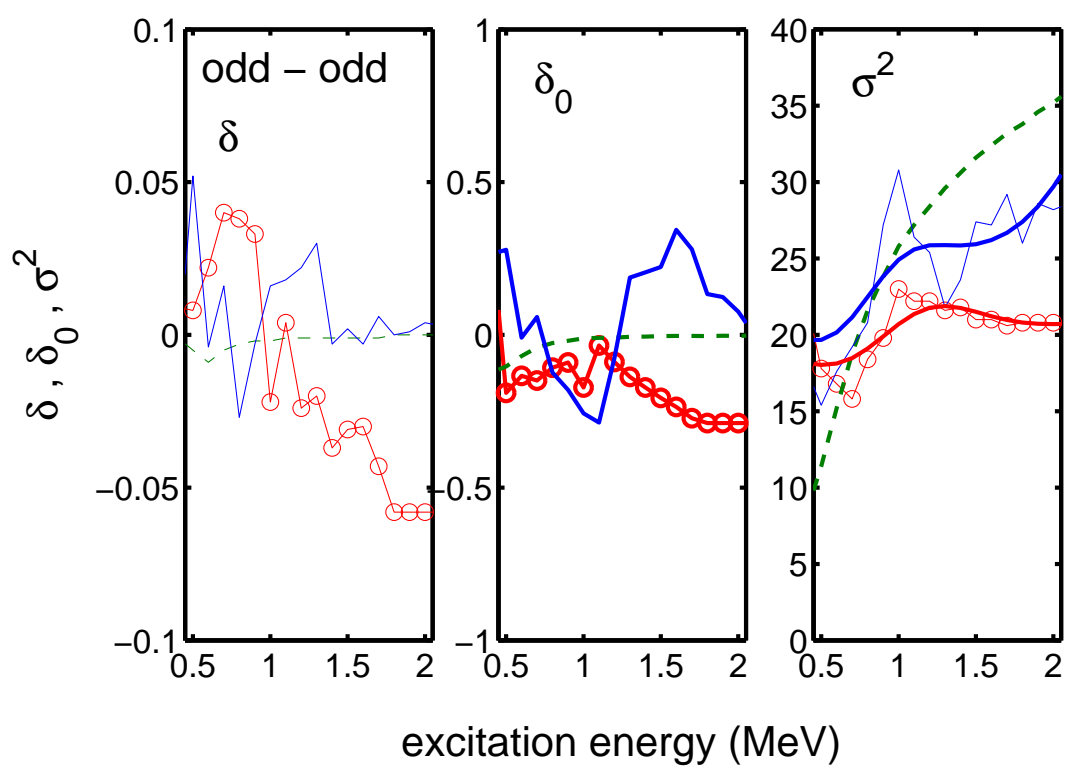

Figure 11: Same as Fig. 9, now for the three odd-odd nuclei of Table 2 (no energy shift for the Fermi gas). The data are incomplete above $0.8 \mathrm{MeV}$.

momentum. However, for the nuclei and values of angular momentum in question, the signature splitting is very small compared to the energy scale over which the cumulative level density is summed. Thus, no signature staggering is observed for odd-mass nuclei $[3,4]$.

\section{Density of $0^{+}$states}

Recent years research have seen a renewed interest in studies of $0^{+}$states in deformed even-even nuclei, see e.g. $[18,19]$. Experimentally the states can be studied to quite high excitation energy with high resolution $(p, t) 2 \mathrm{n}$-transfer reactions. Theoretically, low-lying $0^{+}$states can be excited in terms of collective $\beta$ - or pairing-vibrations (or a mixture of both), or by simple 2-quasi-particle excitations. In the present study vibrational structures are however neglected.

A rotational band is built on each $0^{+}$state. Surveying data for rotational bands in even-even rare-earth nuclei, it is evident that $\mathbf{v}=0$ rotational bands are abundant at low excitation energies. 


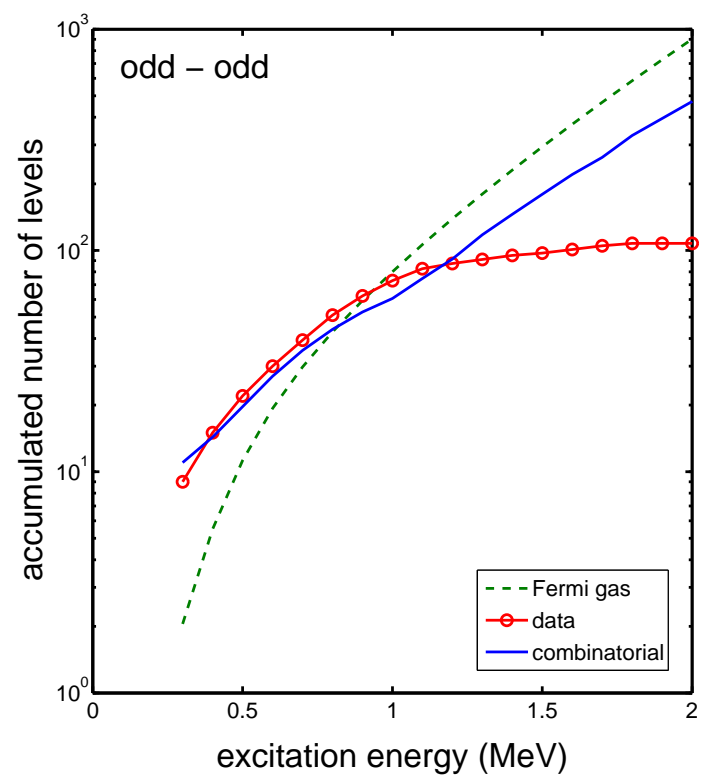

Figure 12: Same as Fig. 8, now for the three odd-odd nuclei of Table2 (no energy shift for the Fermi gas)

In fact, to our knowledge, no $\mathbf{v}>0, K=0^{+}$band containing both even and odd values of the angular momentum has yet been identified.

Performing a careful analysis of pair transfer reactions it is possible to obtain complete level sequences at energies far above $2.1 \mathrm{MeV}$, but with restriction to $I=0^{+}$and $I=2^{+}$states. For the three previously considered even-even nuclei, the most complete data set is found for the nucleus ${ }^{168} \operatorname{Er}[19]$. Although one should take reservations when applying the present model to only one nucleus, it may be interesting to compare the experimental and combinatorial levels for this case. The energy of all $K=0^{+}$band levels below $4 \mathrm{MeV}$ is shown as function of angular momentum in Fig. 13; experimental data in the left-hand frame and calculations in the right-hand frame. In the calculations the ground-state band and the first two excited bands are identified as $\mathbf{v}=0$ bands, while in data one additional $\mathbf{v}=0$ band exists in the same energy interval, $0-2 \mathrm{MeV}$. The first $\mathbf{v}=2$ band is predicted to appear at an excitation energy of about $2.5 \mathrm{MeV}$, and some of the observed $I^{+}=0^{+}$states above $2 \mathrm{MeV}$ are probably of $\mathbf{v}=2$ character. (Actually, in this special case, a $\mathbf{v}=4$ band is predicted at an even lower energy of $2.2 \mathrm{MeV}$ ). It would certainly be very interesting to observe the rotational band on a $K^{\pi}=0^{+}, \mathbf{v}>0$ state, where even as well as odd spin states exist.

The accumulated number of $0^{+}$states for ${ }^{168} \mathrm{Er}$ is shown in fig. 14. The experimental numbers are compared to our calculations, and to two other model calculations including different types of correlations, namely the quasiparticle-phonon model (QPM) $[20,19]$ and the projected shell model (PSM) $[21,19]$. In the present combinatorial model, the levels up to about $2.1 \mathrm{MeV}$ are all twoquasiparticle states, and for these, the model does well in comparison to data, see also Fig. 8. The energy of the more involved four-quasiparticle states, which enter the level density at $2.2 \mathrm{MeV}$, and start to dominate the level density above $3.3 \mathrm{MeV}$, appear to be overestimated, yielding a too low level density. The more involved models, PSM and QPM, with their more complex couplings, are 


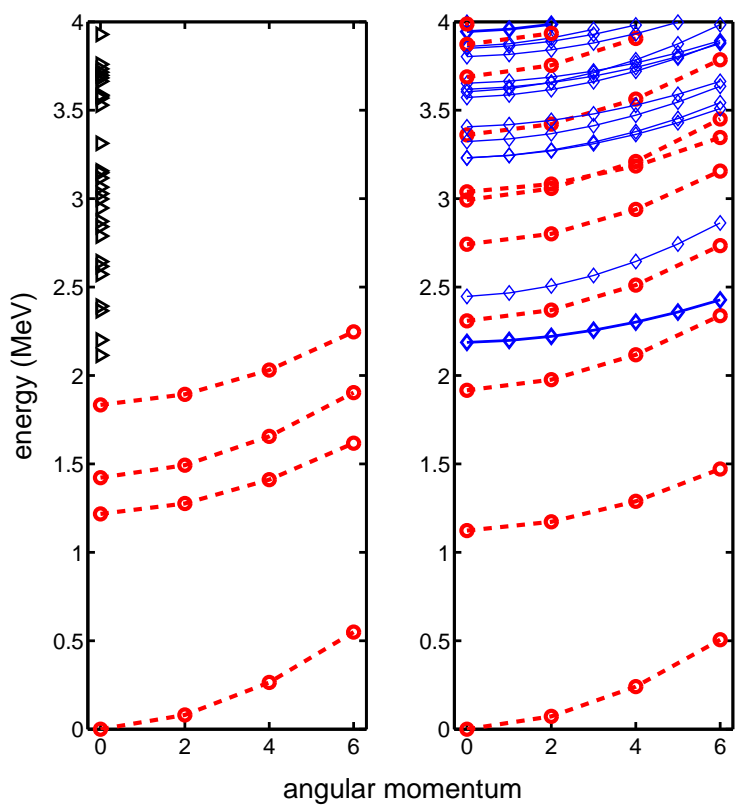

Figure 13: Energy of levels with band-head angular momentum $K=0$ and positive parity for the nucleus ${ }^{168}$ Er as function of angular momentum. Data from the Brookhaven nuclear data base [14] supplied with transfer data [19] are shown in the left-hand frame and levels calculated with the combinatorial level density in the right-hand frame. Seniority $\mathbf{v}=0$ bands are shown by thick red circles connected by dashed lines, and $\mathbf{v}>0$ bands are shown by blue diamonds connected by straight lines. Experimental $I=0$ states not belonging to any observed rotational band are shown by black triangles.

in better agreement with the data at these higher energies.

\section{Other mass regions}

Up to now we have discussed the odd-even staggering in the spin-distribution function for a specific set of well-deformed rare-earth nuclei. However, in the compilation in Ref.[3] the odd-even staggering phenomenon seems to be general, occurring all over the periodic table. This general conclusion is based on summations over wide intervals in mass numbers, containing deformed as well as spherical nuclei. In subsection 5.1 we make a general survey all over the periodic table, based on the Fermi-gas model presented in section 2. In this general survey we ignore that some nuclei are spherical and apply our formalism for deformed nuclei in all regions. And in subsection 5.2 we make a brief study of the odd-even staggering phenomenon in spherical nuclei.

\subsection{Survey applying the Fermi-gas model}

At a first thought, the effect is expected to decrease with increasing mass number, since the level-density parameter $a$ increases linearly with mass number $A$. However, in the analysis by von Egidy and Bucurescu $[3,4]$, the effect was found to be approximately of equal size for all nuclei. In 


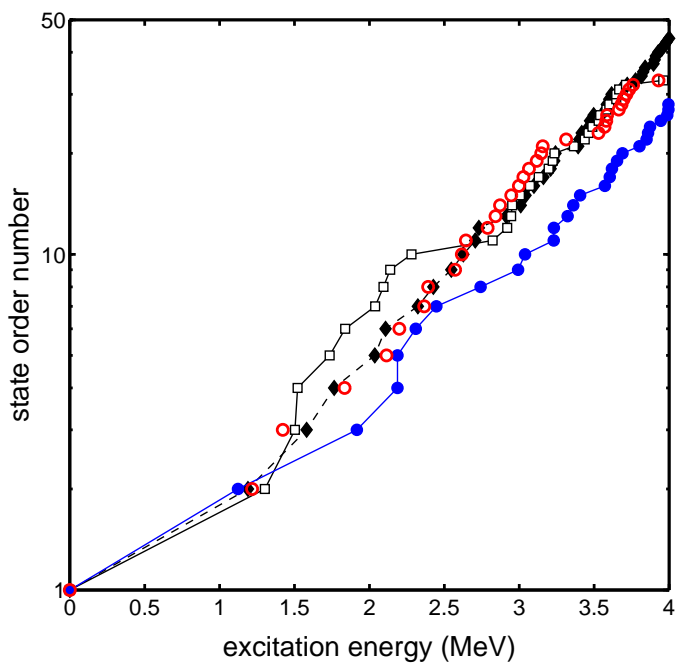

Figure 14: Accumulated number of $I=0^{+}$levels in the nucleus ${ }^{168} \mathrm{Er}$ as function of excitation energy. Data (red circles) - QPM calculations (solid diamonds connected by dashed lines) PSM calculations (open squares connected by solid lines), the current combinatorial calculations (blue filled circles connected by solid lines). The data are from the Brookhaven nuclear data base [14] supplied with transfer data [19], and the results of QPM and PSM calculations are extracted from ref. [19].

this subsection we shall utilize the Fermi gas model to estimate the staggering in the different mass regions, as defined in [3].

A crude estimate of $\delta_{0}$ is represented by the level-density ratio at the maximum energy $E$ of the interval:

$$
\delta_{0} \approx \frac{\rho_{\mathbf{v}=0}(N, Z, E)}{\frac{1}{2} \rho_{\text {intr }}(N, Z, E, K=0)}
$$

In this estimate, one neglects that $\delta_{0}$ is a property of summed levels, and not of the level density itself. Also neglected is the fact that $\mathbf{v}=0$ states contribute as well to the level density at angular momenta $I=2, I=4$ and $I=6$, that also affects the overall normalization. Inserting the Fermi gas expressions (2) and (5), and scaling relative to mass number $A=168$, one obtains:

$$
\delta_{0} \approx\left(\frac{a\left(E-E_{2}\right)}{14}\right)^{\frac{1}{4}}\left(\frac{A}{168}\right)^{\frac{1}{3}} \exp \left(\sqrt{14}-\sqrt{a\left(E-E_{2}\right)}\right)
$$

where the exponential function is the most important. Here, we have applied the calibration from Fig. 9: $\delta_{0} \simeq 1$ for $E-E_{2}=1 \mathrm{MeV}$. Concerning the displacement of the energy $E_{2}$, which is equal to $1.2 \mathrm{MeV}$ at $A=168$, we scale with the mass number to the $-1 / 2$ 'th power, yielding:

$$
E_{2}=\frac{15.6}{\sqrt{A}} \mathrm{MeV}
$$


For the lighter mass number intervals discussed by von Egidy and Bucurescu, the spin window for precise level information only stretches up to $I=4$ for many nuclei, and the ratio between $\delta$ and $\delta_{0}$ should be around $1 / 6$, cf. Eq.((13)). We extracted values of $\sigma^{2}, \delta$ and $\delta_{0}$ fitting the distribution up to $I=4$, and compared to the expression (14) above. The result for $\delta_{0}$ and $\delta$ is displayed inv Table 3.

\begin{tabular}{|l|c|c|c||c|c|c||c|c|}
\hline $\begin{array}{l}\text { mass } \\
\text { interval }\end{array}$ & $\begin{array}{c}\text { number of } \\
\text { states }\end{array}$ & $\langle A\rangle$ & $\langle E\rangle$ & $\begin{array}{c}\delta_{0} \\
\text { analytic }\end{array}$ & $\begin{array}{c}\delta_{0} \\
\text { expt. }\end{array}$ & $\begin{array}{c}\delta \\
\text { expt }\end{array}$ & $\begin{array}{c}\delta_{0} / \delta \\
\text { expt. }\end{array}$ & $\begin{array}{c}\delta_{0} / \delta \\
\text { analytic }\end{array}$ \\
\hline $20-58$ & 569 & 32.5 & 9.05 & 0.41 & 0.54 & 0.20 & 2.7 & 6 \\
\hline $60-98$ & 252 & 81.2 & 3.22 & 1.26 & 1.31 & 0.38 & 3.5 & 6 \\
\hline $100-148$ & 592 & 123.3 & 2.87 & 0.79 & 1.23 & 0.27 & 4.6 & 6 \\
\hline $148-196$ & 655 & 167.8 & 1.99 & 1.42 & 1.40 & 0.26 & 5.4 & 6 \\
\hline $200-250$ & 365 & 228.1 & 2.22 & 0.45 & 0.63 & 0.20 & 3.1 & 6 \\
\hline
\end{tabular}

Table 3: Staggering parameters for even-even nuclei are given for five different mass intervals. Columns 3 and 4 give the average mass number and average upper excitation energy to which level counts are considered accurate, according to reference [4]. The analytic values of $\delta_{0}$ are calculated with the crude Fermi-gas approximation (14). The experimental values of $\delta_{0}$ and $\delta$ are obtained from fits to the curves given in reference [3]. Finally, the two last columns compare the ratio $\delta_{0} / \delta$ to the relation $\delta_{0} / \delta=\left(I_{\max }+2\right)=6$, providing a crude approximation for deformed nuclei.

With increasing mass number through the different mass intervals, the upper energy for sampling the states decreases while the level-density constant increases. These two effects work in different directions on the expression in the exponential of equation (14). As a result, the obtained overabundance of $I=0$ states, $\delta_{0}$ appears to be rather constant through the mass table, with values between 0.5 and 1.4 .

For a given mass number, the relation $\delta_{0}=1$ marks the boundary between low energies, where $\mathbf{v}=0$ states dominate the $K=0$ bandheads and higher energies, where $\mathbf{v}>0$ states dominate. A good approximation to (14) for $\delta_{0}=1$ is given by the simple expression

$$
E^{*} \approx 61 A^{-0.65} \mathrm{MeV}
$$

which is displayed in Fig. 15. The figure shows that the energies approximately follow this line, which roughly explains the approximate constancy of $\delta_{0}$ through the mass table. The dominance of $\mathbf{v}=0$ states quickly disappears as excitation energy increases above $E^{*}$. For comparison, the boundary where the density of $\mathbf{v}=0$ states are half the density of $\mathbf{v}>0$ states, i.e. $\delta_{0}=1 / 2$ in Eq.(14), is shown by a dashed line in Fig. 15.

\subsection{Odd-even staggering in spherical nuclei}

In the left-hand part of Fig. 16 we show spin-distribution functions obtained from observed spin states in eight spherical nuclei in the mass region 100-148, and in the right-hand part three welldeformed nuclei from the same mass region. A clear odd-even staggering is seen for the spherical nuclei that is very similar to the deformed nuclei. This observation is in line with the theoretical calculation in Ref.[2] where an odd-even staggering was found in the spherical nucleus ${ }^{56} \mathrm{Fe}$. All these spherical cases are indeed open-shell nuclei. The staggering in the only two closed-shell 


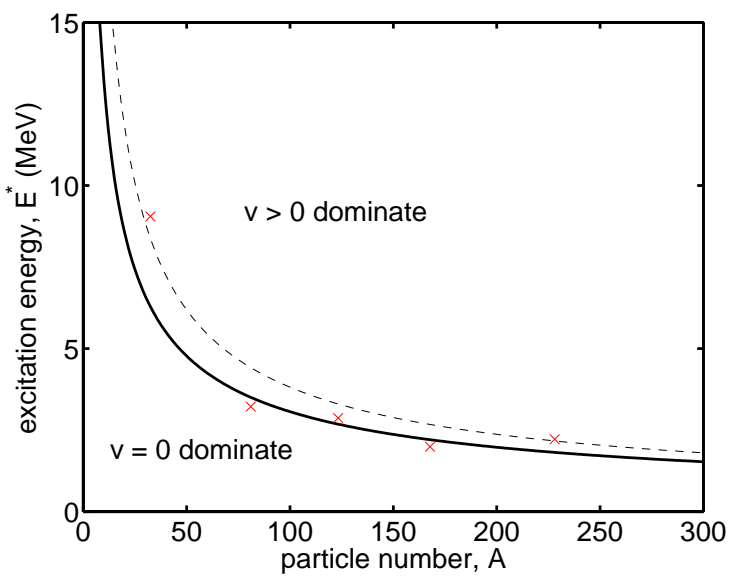

Figure 15: The approximate boundary for $\mathbf{v}=0$ states, $E^{*} \approx 61 A^{-0.65} \mathrm{MeV}$ is displayed by a solid line together with the present experimental upper limits of excitation energy (red crosses). The dashed line shows the boundary defined by $\delta_{0}=1 / 2$ in Eq.(14).

spherical nuclei in the compilation is shown in fig.17. One should not draw too firm conclusions on the basis of data from only two nuclei. However, a completely different picture emerges with no systematic odd-even staggering. In particular angular momentum I=3 states are relatively more abundant as compared to open-shell nuclei.

We thus see that open-shell spherical nuclei consistently seem to exhibit an odd-even staggering in the spin distribution function, favouring even spins, while the spin distribution in closed-shell spherical nuclei is more irregular. Clearly, the odd-even staggering in spherical nuclei has a different origin than in deformed nuclei, where, as here discussed, the $r$-symmetry of the quadrupole deformed nucleus is the explanation. In spherical nuclei the origin of the staggering can be related to the fermion exchange symmetry: e.g. two equal particles in a $j$-shell can couple to the spin values, $I=0,2, . .2 j-1$. On the other hand, in closed-shell spherical nuclei states are based on particlehole excitations across the shell gap creating configurations in different $j$-shells, implying no special odd-even selection with respect to angular momentum.

\section{Conclusions and Summary}

We have investigated the role of seniority-zero states in level densities of deformed nuclei. Analytical expression were derived for the Fermi-gas level density, and the $\mathbf{v}=0$ density was compared to the density of $\mathbf{v}>0$ states. Due to symmetries of the deformed nuclear potential the $\mathbf{v}=0$ states give rise to even angular momentum states only, implying possibilities for an odd-even staggering of the spin-distribution function. Such a staggering behavior was recently observed [3, 4], and we could provide a detailed theoretical explanation of the phenomenon. Indeed, a simple explanation based on analytical expressions was given why $\mathbf{v}=0$ states dominate at low excitation energies while they play no role at higher excitation energies. Incidentally, the present energy limit for identifying discrete states in even-even nuclei is found to correspond roughly to the transition energy from $\mathbf{v}=0$ to $\mathbf{v}=2$ dominance of the calculated number of levels at angular momentum 


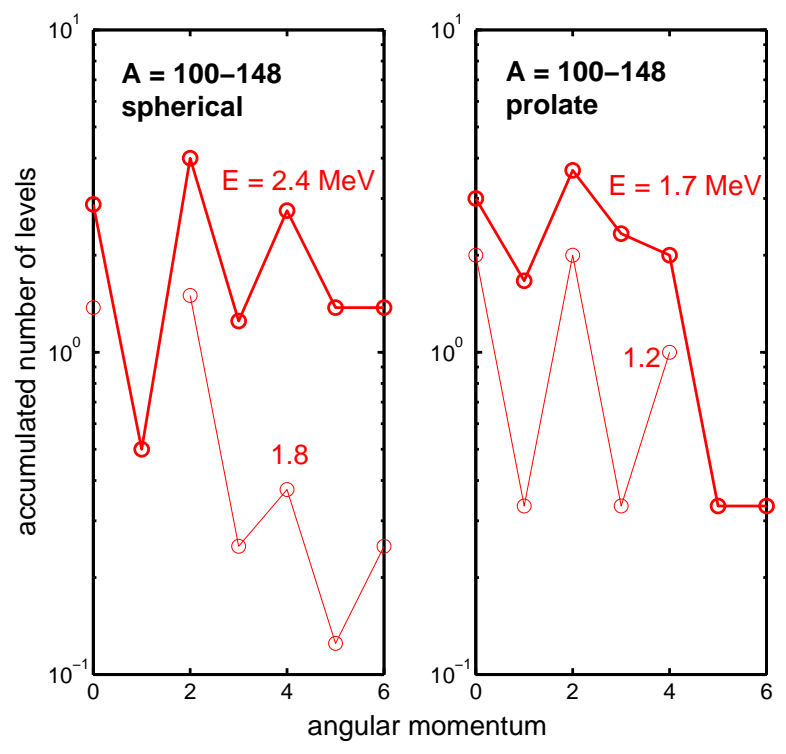

Figure 16: Spin-distributions from observed energy levels in a set of spherical (left-hand part) and deformed (righthand part) nuclei in the mass region $\mathrm{A}=100-148$. The spherical nuclei are ${ }_{50}^{116,118,120} \mathrm{Sn}, \frac{132}{54} \mathrm{Xe},{ }_{56}^{136,138} \mathrm{Ba},{ }_{58}^{142} \mathrm{Ce}$, ${ }_{60}^{144} \mathrm{Nd}$

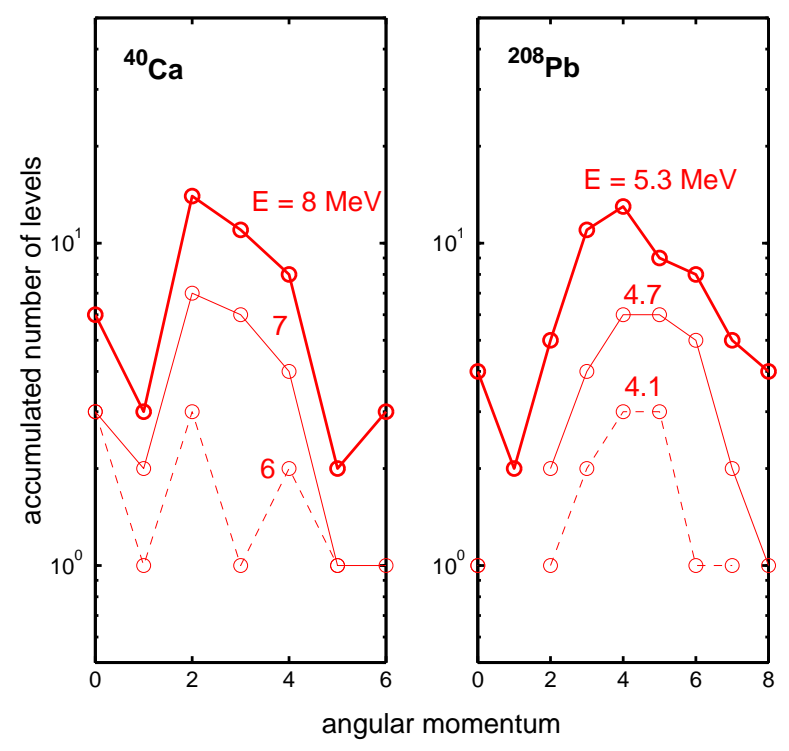

Figure 17: Spin-distribution for a compilation obtained from experimental data for the two closed-shell nuclei, ${ }_{20}^{40} \mathrm{Ca}$ and ${ }_{82}^{208} \mathrm{~Pb}$ 
$I=0$. Consequently, all (observed) deformed even-even nuclei are expected to display an odd-even staggering in the level density, with staggering parameters $\delta_{0} \approx 1, \delta \approx 1 / 6$.

In references $[3,4]$, the odd-even staggering was discussed as a pairing effect, while for the deformations here considered, we show that the staggering also appears for an unpaired Fermi gas, provided the potential is deformed and carries $r$-symmetry.

Quantitative comparisons between the combinatorial level-density model and data were performed for (i) low-energy level densities for three even-even and three odd-odd nuclei, and (ii) the density of $0^{+}$states in ${ }^{168} \mathrm{Er}$ up to the quite high excitation energy of $4 \mathrm{MeV}$. Up to about $2.1 \mathrm{MeV}$ in even-even and to $0.8 \mathrm{MeV}$ in odd-odd nuclei, we find an excellent agreement between calculations and data, both for the summed level density, and the angular momentum dependence. Within these energy intervals, only 2 quasiparticle excitations are found in the calculations. Especially, for $I^{\pi}=0^{+}$, only $\mathbf{v}=0$ states are found. Although one should be careful concerning the comparison to just one dataset for one nucleus, it appears that the combinatorial level density underpredicts the level density within the excitation energy interval from 3 to $4 \mathrm{MeV}$, where four-quasiparticle levels dominate the level density. This may be due to lack of vibrational couplings in the model. It would be interesting to have the same type of data for more nuclei.

A brief discussion was given of the odd-even staggering phenomenon all over the periodic table. Qualitatively the here developed Fermi-gas model was able to give an over all account of the mass-dependence. Finally, odd-even staggering in spherical nuclei was discussed, where odd-even staggering phenomenon appears in open-shell nuclei, while closed-shell nuclei exhibit an irregular behaviour. In spherical nuclei the odd-even staggering in the spin-distribution function is caused by the fermion exchange symmetry.

\section{Acknowledgments}

S. Å. and B.G.C. thank the Swedish Natural Science research council (VR) for support. P.M. thanks the division of Mathematical Physics, Lund University, for hospitality during several visits. The work of P.M. was carried out under the auspices of the National Nuclear Security Administration of the US Department of Energy at Los Alamos National Laboratory under Contract No. DE-AC52-06NA25396.

\section{References}

[1] A.C. Larsen et. al., Phys. Rev. C 83 (2011) 034315, and references therein.

[2] Y. Alhassid, S. Liu and H. Nakada, Phys. Rev. Lett. 99, 162504 (2007).

[3] T. von Egidy and D. Bucurescu, Phys. Rev. C 78 (2008) 051301 (R).

[4] T. von Egidy and D. Bucurescu, Phys. Rev. C 80 (2009) 054310.

[5] P. Möller, A.J. Sierk, T. Ichikawa, R. Bengtsson H. Uhrenholt and S. Åberg, Phys. Rev. C79 (2009) 064304.

[6] P. Möller, J.R. Nix, W.D. Myers and W.J. Swiatecki, At. Data Nucl. Data Tables 59 (1995) 185.

[7] L. Bonneau, P. Quentina and P. Möller, Phys. Rev. C76 (2007) 024320. 
[8] P. Möller, J.R. Nix, K.-L- Kratz, At. Data Nucl. Data Table 66 (1997) 131.

[9] S. Hilaire and S. Goriely, Nucl. Phys. A 779 (2006) 63.

[10] H. Uhrenholt, S. Åberg, A. Dobrowolski, T. Døssing, T. Ichikawa and P. Möller, Nucl. Phys. A 913 (2013) 127. 104.

[11] A. Bohr and B. R. Mottelson, Nuclear Structure, Volume I (Benjamin, Reading, MA, 1969), Appendix 2B.

[12] R. Bengtsson and S. Åberg, Phys. Lett. B 172 (1986) 277.

[13] A. Bohr and B. R. Mottelson, Nuclear Structure, Volume 2 (Benjamin, Reading, MA, 1975).

[14] Brookhaven Nuclear Database, www.nndc.bnl.gov.

[15] B. Lauritzen, T. Døssing and R. A. Broglia, Nucl. Phys. A 457 (1986) 61.

[16] T. Døssing, T.L. Khoo, T. Lauritsen, I. Ahmad, D. Blumenthal, M.P. Carpenter, B. Crowell, D. Gassmann, R.G. Henry, R.V.F. Janssens, D. Nisius, Phys. Rev. Lett. 75 (1995) 1276

[17] H. T. Nyhuus, S. Siem, M. Guttormsen, A.C. Larsen, A Bürger, N.U.H.Syed, H.K. Toft, G.M. Tveten and A. Voinov, Phys. Rev. C85 (2012) 014323

[18] P.E. Garrett, J. Phys. G: Nucl. Part. Phys. 27, R1 (2001).

[19] D. Bucurescu, G. Graw, R. Hertenberger, H.-F. Wirth, N. Lo Iudice, A.V. Sushkov, N. Yu. Shirikova, Y. Sun, T. Faestermann, R. Krücken, M. Mahgoub, J. Jolie, P. von Brentano, N. Braun, S. Heinze, O. Möller, D, Müncher, C. Scholl, R.F. Casten and D. A. Meyer, Phys. Rev. C 73 (2006) 064309.

[20] V.G. Soloviev, Theory of Atomic Nuclei: Quasiparticles and Phonons (Institute of Physics, Bristol, 1992).

[21] K. Hara and Y. Sun, Int. J. Mod.Phys. E 4, 637 (1997). 